University of Nebraska - Lincoln

DigitalCommons@University of Nebraska - Lincoln

8-22-1999

\title{
A patching model for surface tension and the Tolman length
}

T.V. Bykov

University of Nebraska-Lincoln

Xiao Cheng Zeng

University of Nebraska-Lincoln, xzeng1@unl.edu

Follow this and additional works at: https://digitalcommons.unl.edu/chemzeng

Part of the Chemistry Commons

Bykov, T.V. and Zeng, Xiao Cheng, "A patching model for surface tension and the Tolman length" (1999). Xiao Cheng Zeng Publications. 58.

https://digitalcommons.unl.edu/chemzeng/58

This Article is brought to you for free and open access by the Published Research - Department of Chemistry at DigitalCommons@University of Nebraska - Lincoln. It has been accepted for inclusion in Xiao Cheng Zeng Publications by an authorized administrator of DigitalCommons@University of Nebraska - Lincoln. 


\title{
A patching model for surface tension and the Tolman length
}

\author{
T. V. Bykov and X. C. Zeng \\ Department of Chemistry, University of Nebraska-Lincoln, Lincoln, Nebraska 68588
}

(Received 15 April 1999; accepted 28 May 1999)

In the framework of density functional theory (DFT), a patching model for the density profile of the
liquid-vapor interface is developed. The patching is based on analytical expressions of the
asymptote of the density profiles. Derived from the model the surface tension of planar liquid-vapor
interface as well as the Tolman length can be computed from analytic expressions. Two prototype
systems are considered; the Yukawa and the Lennard-Jones. As a result, the temperature
dependence of the surface tension as well as the Tolman length are obtained. The results are
compared with numerical DFT calculations. (c) 1999 American Institute of Physics. [S0021-9606(99)70232-1]

\section{INTRODUCTION}

Surface tension is one of the fundamental characteristics of both a planar liquid-vapor interface and a liquid droplet. The difference between the two when the droplet is large can be accounted for by the Tolman correction, namely, the Tolman length divided by the radius of the droplet. ${ }^{1}$ In the classical homogeneous nucleation theory, ${ }^{2}$ the surface tension is essentially an input parameter which is usually taken from experiments. On the other hand, the surface tension can also be determined by means of statistical mechanics, for example, molecular dynamics and Monte Carlo simulation methods ${ }^{3}$ or the density functional theory. ${ }^{4}$

The molecular dynamics simulation has been used to calculate the surface tension of relatively small liquid droplets which contains a few hundreds of particles. ${ }^{5}$ Values of the Tolman length were computed for such liquid droplets, which were typically a few molecular diameters. However, because these droplets are so small that not the Tolman correction, but the higher order curvature corrections dominate the behavior of surface tension of the droplets, ${ }^{6}$ and the effect of so-called self-overlapping of surface layer takes place. ${ }^{7}$ Therefore, it is practically impossible to obtain the true value of the Tolman length for such small droplets. Molecular dynamics simulations for larger systems (tens of thousand particles) have also been carried out. ${ }^{8,9}$ Values of the Tolman length were found to be either nearly zero ${ }^{8}$ or positive but very small. ${ }^{9}$ Although it is possible to simulate even larger droplets (e.g., with millions of particles) there is still a size limit in the simulations.

An alternative method to calculate the surface tension and the Tolman length is the density functional theory (DFT). Although DFT is only semiquantitative compared to the molecular dynamics simulation, it allows us to calculate the surface tension and the Tolman length in the limit of infinitely large droplet. Using DFT, several groups have studied the dependence of surface tension on curvature for spherical droplets. ${ }^{10,11,7}$ In all studies negative values of the Tolman length are found. Another density functional approach was recently developed by Blokhuis and Bedeaux. ${ }^{12}$ This approach is based on the decomposition of the density profile in small curvature of the droplet. They showed that the surface tension and curvature coefficients can be calculated by using statistical mechanics formulas, such as the Kirkwood-Buff formula for surface tension, when the density profile for planar liquid-vapor interface and its curvature corrections are known. They also showed in a later work $^{13}$ that in the most simple case of the gradient approximation for the free energy density, these statistical mechanics formulas reduce to mean-field formulas, originally derived by Fisher and Wortis. ${ }^{14}$ Fisher and Wortis also found negative values for the Tolman length based on the expansion of free energy density near the critical point. When more sophisticated numerical approaches were taken, ${ }^{15,6}$ again, negative values for the Tolman length were obtained. Temperature dependence of the surface tension, the Tolman length, and effective rigidity constant were also studied in these works.

Thus far, all reported calculations of the temperature dependence of the Tolman length relied on some numerical methods, e.g., numerical iteration and integration, and therefore only numerical results were obtained. It would be desirable and useful to have analytical results for the surface tension and the Tolman length. A few attempts have been made along this line. ${ }^{16,17}$ In this work, we will develop an approach to derive analytical results for the surface tension and the Tolman length in the framework of density functional theory. Key to this approach is a sensible analytical approximation for the density profile, which can be used in the statistical mechanics formulas for the surface tension and the Tolman length. We call our approach the patching model for the asymptote of density profile.

The remainder of the paper is organized in the following way. In Sec. II the general equation for asymptote of density profile is derived. Sections III and IV give the solution of this equation for Yukawa and Lennard-Jones systems, respectively. The resulting analytical expressions of density profile of the planar interface are also presented. In Sec. V analytical formulas for the surface tension and the Tolman length are derived, and results are compared with the accurate numerical calculations. The conclusion is given in Sec. VI. 


\section{THE EQUATION FOR THE ASYMPTOTE OF DENSITY PROFILE}

In the framework of density functional theory the free energy of the liquid-vapor system with density profile $\rho(\mathbf{r})$ can be written as ${ }^{4}$

$$
\begin{aligned}
F[\rho]= & F_{\mathrm{id}}[\rho]+F_{\mathrm{ex}}\left[\rho_{b}\right]-k_{B} T \int d \mathbf{r} \Delta \rho(\mathbf{r}) c^{(1)}\left(\rho_{b}\right) \\
& +k_{B} T \int_{0}^{1} d \alpha(\alpha-1) \int d \mathbf{r}^{\prime} \int d \mathbf{r}^{\prime \prime} \Delta \rho\left(\mathbf{r}^{\prime}\right) \\
& \times \Delta \rho\left(\mathbf{r}^{\prime \prime}\right) c^{(2)}\left(\left[\rho_{\alpha}\right] ; \mathbf{r}^{\prime}, \mathbf{r}^{\prime \prime}\right) .
\end{aligned}
$$

Here $F_{\text {id }}[\rho]$ is the ideal gas contribution to the free energy, $\rho_{b}$ is the density in the bulk phase, $F_{\text {ex }}\left[\rho_{b}\right]$ is the excess free energy of the bulk phase over that of the ideal gas, and $\rho_{\alpha}$ has the form $\rho_{\alpha}=\rho_{b}+\alpha \Delta \rho$, where $\Delta \rho=\rho(\mathbf{r})-\rho_{b}$. In Eq. (1) $c^{(1)}\left(\rho_{b}\right)$ and $c^{(2)}\left(\left[\rho_{\alpha}\right] ; \mathbf{r}^{\prime}, \mathbf{r}^{\prime \prime}\right)$ are the first and the second direct correlation function of the system.

The grand potential of such system with chemical potential $\mu$ is related to the free energy through

$$
\Omega[\rho]=F[\rho]-\mu \int d \mathbf{r} \rho(\mathbf{r}),
$$

from which the equilibrium density profile can be determined using the variation condition,

$$
\frac{\delta \Omega[\rho]}{\delta \rho}=0 .
$$

Combining Eqs. (1)-(3) leads to the equation ${ }^{4}$

$$
\begin{aligned}
& \ln \frac{\rho\left(\mathbf{r}^{\prime}\right)}{\rho_{b}}+2 \int_{0}^{1} d \alpha(\alpha-1) \int d \mathbf{r}^{\prime \prime} c^{(2)}\left(\left[\rho_{\alpha}\right] ; \mathbf{r}^{\prime}, \mathbf{r}^{\prime \prime}\right) \Delta \rho\left(\mathbf{r}^{\prime \prime}\right) \\
& \quad+\int_{0}^{1} d \alpha(\alpha-1) \alpha \int d \mathbf{r}^{\prime \prime} \int d \mathbf{r}^{\prime \prime \prime} c^{(3)}\left(\left[\rho_{\alpha}\right], \mathbf{r}^{\prime}, \mathbf{r}^{\prime \prime}, \mathbf{r}^{\prime \prime \prime}\right) \\
& \quad \times \Delta \rho\left(\mathbf{r}^{\prime \prime}\right) \Delta \rho\left(\mathbf{r}^{\prime \prime \prime}\right)=0,
\end{aligned}
$$

where $c^{(3)}\left(\left[\rho_{\alpha}\right] ; \mathbf{r}^{\prime}, \mathbf{r}^{\prime \prime}, \mathbf{r}^{\prime \prime \prime}\right)$ is the third direct correlation function of the system. Equation (4) is the general form for the equilibrium density profile. To derive the equation for the asymptote of density profile at large absolute values of $\mathbf{r}^{\prime}$, one can assume that in the region near the bulk $\Delta \rho\left(\mathbf{r}^{\prime}\right) / \rho_{b}$ is a small quantity. Therefore, the next two expansions are valid,

$$
\begin{aligned}
c^{(2)}\left(\left[\rho_{\alpha}\right] ; \mathbf{r}^{\prime}, \mathbf{r}^{\prime \prime}\right)= & c^{(2)}\left(\rho_{b}, r\right) \\
& +\left.\alpha \Delta \rho\left(\mathbf{r}^{\prime}\right) \frac{\partial c^{(2)}(\rho, r)}{\partial \rho}\right|_{\rho=\rho_{b}}+\cdots
\end{aligned}
$$

and

$\ln \frac{\rho\left(\mathbf{r}^{\prime}\right)}{\rho_{b}}=\ln \left(1+\frac{\Delta \rho\left(\mathbf{r}^{\prime}\right)}{\rho_{b}}\right)=\frac{\Delta \rho\left(\mathbf{r}^{\prime}\right)}{\rho_{b}}-\frac{1}{2}\left(\frac{\Delta \rho\left(\mathbf{r}^{\prime}\right)}{\rho_{b}}\right)^{2}+\cdots$,

where $r=\left|\mathbf{r}^{\prime}-\mathbf{r}^{\prime \prime}\right|$ and $c^{(2)}\left(\rho_{b}, r\right)$ is the second direct correlation function of the uniform bulk phase. Keeping only terms of the first order in Eq. (4) and using integration by parts, one can obtain the equation for the asymptote of density profile,

$$
\Delta \rho\left(\mathbf{r}^{\prime}\right)=\rho_{b} \int d \mathbf{r}^{\prime \prime} c^{(2)}\left(\rho_{b}, r\right) \Delta \rho\left(\mathbf{r}^{\prime \prime}\right) .
$$

For a planar liquid-vapor interface with density profile $\rho(z)$ Eq. (7) takes the form

$$
\Delta \rho(z)=2 \pi \rho_{b} \int_{0}^{\infty} r^{2} d r \int_{-1}^{1} c^{(2)}\left(\rho_{b}, r\right) \Delta \rho(z+s r) d s,
$$

where $z$ is the distance from the dividing surface. Equation (8) can be further written as

$\frac{d \Delta \rho(z)}{d z}=2 \pi \rho_{b} \int_{0}^{\infty} r d r c^{(2)}\left(\rho_{b}, r\right)(\Delta \rho(z+r)-\Delta \rho(z-r))$.

Having derived Eq. (9) we need to employ appropriate free energy functional in order to derive the second direct correlation function appeared in Eq. (9). In this work we consider two systems, Yukawa and Lennard-Jones, both can be treated as hard-spheres plus a small attractive potential $w(r)$. For both systems we adopt the free energy functional with the local density approximation for the hard-spheres and random phase approximation for the attractive part. ${ }^{4,18}$ The resulting second direct correlation function takes the form

$c^{(2)}\left(\rho_{b}, r\right)=\frac{\delta(r)}{\rho_{b}}-\frac{1}{k_{B} T} \frac{\partial \mu_{h}}{\partial \rho}\left(\rho_{b}\right) \delta(r)-\frac{1}{k_{B} T} w(r)$,

where $\delta(r)$ is the Dirac delta function and $\mu_{h}(\rho)$ is the chemical potential of hard-spheres in Carnahan-Starling form. ${ }^{4,18}$ Substituting Eq. (10) into Eq. (9) yields

$$
\begin{gathered}
\frac{\partial \mu_{h}}{\partial \rho}\left(\rho_{b}\right) \frac{d \Delta \rho(z)}{d z}+2 \pi \int_{0}^{\infty} d r r w(r)(\Delta \rho(z+r) \\
-\Delta \rho(z-r))=0 .
\end{gathered}
$$

Equation (11) is a key result of this work. Analytical solutions of Eq. (11) for two prototypical fluid systems, Yukawa and Lennard-Jones, are given in the next two sections.

\section{THE MODEL OF THE DENSITY PROFILE FOR A SYSTEM WITH SHORT-RANGE POTENTIAL}

As a model of short-range potential the attractive part of the Yukawa potential has the form

$$
w(r)=-\frac{\beta \lambda^{3}}{4 \pi} \frac{\exp (-\lambda r)}{\lambda r},
$$

where $\lambda=1 / d$ ( $d$ is the hard-sphere diameter) and $\beta=$ $-4 \pi \int_{0}^{\infty} d r r^{2} w(r)$ is a parameter of the potential. We assume that for the short-range potential the asymptote of density profile has the exponential form ${ }^{4,16}$

$$
\Delta \rho(z)=c_{b} \exp (\zeta \lambda z),
$$

where $c_{b}$ and $\zeta$ are two undeterminate parameters. Substituting Eq. (13) into Eq. (11) results in 


$$
\frac{\partial \mu_{h}}{\partial \rho}\left(\rho_{b}\right)+4 \pi \int_{0}^{\infty} d r r^{2} w(r) \frac{\sinh (\zeta \lambda r)}{\zeta \lambda r}=0 .
$$

The integral in Eq. (14) can be carried out easily if the $\zeta$ parameter in Eq. (13) is less than 1. Indeed, we find that only such values of $\zeta$ are physically meaningful; otherwise, the density profile would change significantly for $z$ much larger than $d$. Under the aforementioned precondition the solution of Eq. (14) can be written analytically as

$$
\begin{aligned}
\zeta\left(\rho_{b}\right) & =\left(\frac{\partial \mu}{\partial \rho}\left(\rho_{b}\right)\right)^{1 / 2}\left(\frac{\partial \mu_{h}}{\partial \rho}\left(\rho_{b}\right)\right)^{-1 / 2} \\
& =\left(\rho_{b}^{2} \chi_{b} \frac{\partial \mu_{h}}{\partial \rho}\left(\rho_{b}\right)\right)^{-1 / 2},
\end{aligned}
$$

where $\chi_{b}$ is the compressibility of the bulk phase. In deriving Eq. (15) we also used the relation $(\partial \mu / \partial \rho)\left(\rho_{b}\right)=\left(\rho_{b}^{2} \chi_{b}\right)^{-1}$ $=\left(\partial \mu_{h} / \partial \rho\right)\left(\rho_{b}\right)-\beta$. It is easy to see that Eq. (15) always leads to $\zeta<1$, which is consistent with the precondition required to solve Eq. (14). In passing we point out that if one uses a simple gradient expansion for the free energy functional $^{4}$ and keeps only the first square gradient term, one will find that values of $\zeta$ are greater than 1 for most temperatures (except near the critical point), which in turn leads to a large gradient of the density profile. Consequently, not only the square but also higher order gradient terms should be taken into account. Thus the square gradient approximation works well only near the critical point.

To proceed the derivation we rewrite Eq. (13) in a more specific form,

$$
\begin{aligned}
& \rho(z)=\rho_{1}+c_{1} \exp \left(-\zeta_{1} \lambda z\right), \quad z>z_{0}, \\
& \rho(z)=\rho_{2}-c_{2} \exp \left(\zeta_{2} \lambda z\right), \quad z<z_{0},
\end{aligned}
$$

where $\rho_{1}$ and $\rho_{2}$ are, respectively, the density of the uniform vapor and liquid, $\zeta_{1}=\zeta\left(\rho_{1}\right)$ and $\zeta_{2}=\zeta\left(\rho_{2}\right)$. It turns out that Eq. (16) describes the density profile very well near bulk regions where the condition $\Delta \rho(z) / \rho_{b}<1$ is satisfied. This condition is essential for deriving Eq. (9). In particular, this condition works for $z<z_{0}$ except for a small region near $z_{0}$, where $z>z_{0}$.

Three unknown parameters in Eq. (16) are $c_{1}, c_{2}$, and $z_{0}$. To obtain $c_{1}$ and $c_{2}$ a simple patching model is devised. Specifically, the conditions of continuity of density profile $\rho(z)$ and its derivation $(\partial \rho(z) / \partial z)$ at $z_{0}$ yield

$$
\begin{aligned}
& c_{1}=\left(\rho_{2}-\rho_{1}\right) \frac{\zeta_{2}}{\zeta_{1}+\zeta_{2}} \exp \left(\zeta_{1} \lambda z_{0}\right), \\
& c_{2}=\left(\rho_{2}-\rho_{1}\right) \frac{\zeta_{1}}{\zeta_{1}+\zeta_{2}} \exp \left(-\zeta_{2} \lambda z_{0}\right) .
\end{aligned}
$$

Value of $z_{0}$ depends on the choice of dividing surface. Here we used the equimolar dividing surface (located at $z=0$ ), which is defined by the equation

$$
\int_{-\infty}^{\infty} z \frac{\partial \rho(z)}{\partial z} d z=0
$$

Equations (16) and (18) give forth

$$
z_{0}=d \frac{\zeta_{1}-\zeta_{2}}{\zeta_{1} \zeta_{2}}
$$

Having derived the analytical expressions for $c_{1}, c_{2}$, and $z_{0}$ [Eqs. (17) and (19)] the patching model for the density profile of Yukawa liquid-vapor interface is fully established.

\section{THE MODEL OF THE DENSITY PROFILE FOR A SYSTEM WITH LONG-RANGE POTENTIAL}

A widely studied long-range potential is the LennardJones. In this case the attractive part of potential $w(r)$ can be expressed in the form introduced by Weeks, Chandler, and Anderson $^{19}$ as

$$
\begin{aligned}
& w(r)=-\epsilon, \quad r<r_{0}, \\
& w(r)=4 \epsilon\left(\left(\frac{\sigma}{r}\right)^{12}-\left(\frac{\sigma}{r}\right)^{6}\right), \quad r>r_{0} .
\end{aligned}
$$

Here $r_{0}=2^{1 / 6} \sigma$, and $\epsilon$ and $\sigma$ are parameters of the LennardJones potential. Temperature-dependent hard-sphere diameter is related to the parameter $\sigma$ through $d(T)=\left(a_{1} T\right.$ $+b) /\left(a_{2} T+a_{3}\right) \sigma .{ }^{18}$ Values of the constants $a_{1}, a_{2}, a_{3}$, and $b$ are taken from Ref. 18 .

Unlike the Yukawa system, it is found that for LennardJones the exponential form as Eq. (13) for the asymptote of the density profile is no longer valid. This is because the integral in Eq. (14) would be divergent if $w(r)$ was given as Eq. (20). Clearly, a new function form for the asymptote has to be determined first. Moreover, if one takes a gradient expansion for the free energy and keeps only the first square gradient term, one would obtain solutions in form of Eq. (13). But the problem would be that only the first coefficient in the square gradient expansion is finite and all other coefficients diverge for Lennard-Jones. One possible solution to the problem is to use an alternative interaction potential such that it is zero beyond a certain distance. ${ }^{20}$ Another way to solve this problem, which is taken in this work, is to find a new function form for the asymptote of density profile.

To obtain the correct asymptote for the solution of Eq. (11) we shall use a Fourier transformation technique, originally suggested by Evans and co-workers. ${ }^{21}$ We applied Eq. (11) in vapor region $\left(z>z_{1}\right)$ and liquid region $\left(z<z_{2}\right)$, where $z_{1}$ and $z_{2}$ are boundary points for which the validity of Eq. (11) was examined. Let us first consider the solution of Eq. (11) for $\rho_{b}=\rho_{1}$ (situation for $\rho_{b}=\rho_{2}$ can be described similarly). The solution can be generally written as

$$
\begin{aligned}
& \Delta \rho(z)=0, \quad z<z_{1}, \\
& \Delta \rho(z)=\Delta \rho_{1}(z), \quad z>z_{1},
\end{aligned}
$$

where $\Delta \rho_{1}(z)$ is the target function we intend to derive. Equation (11) can be rewritten as

$$
m_{1} g(z)+2 \pi \int_{0}^{\infty} d r r w(r)[\Delta \rho(z+r)-\Delta \rho(z-r)]=0,
$$

where $m_{1}=\left(\partial \mu_{h} / \partial \rho\right)\left(\rho_{1}\right)$ and $g(z)=(d \Delta \rho(z) / d z)$. The Fourier transformation of $g(z)$ is 


$$
g(q)=\int_{-\infty}^{\infty} d z \exp (-i q z) g(z)
$$

and that of $w(r)$ is

$$
w(q)=4 \pi \int_{0}^{\infty} d r r^{2} w(r) \frac{\sin q r}{q r} .
$$

Taking the Fourier transformation of Eq. (22) yields

$$
m_{1} g(q)+w(q) \int_{-\infty}^{\infty} d z(i q) \exp (-i q z) \Delta \rho(z)=0 .
$$

Substituting Eq. (21) into Eq. (25) and using integral by parts produces

$$
m_{1} g(q)+w(q)\left(-c_{1} \exp \left(-i q z_{1}\right)+g(q)\right)=0,
$$

where $c_{1}=-\Delta \rho_{1}\left(z_{1}\right)$. The solution of Eq. (26) is simply

$$
g(q)=\frac{c_{1} w(q) \exp \left(-i q z_{1}\right)}{m_{1}+w(q)} .
$$

Taking the inverse Fourier transformation of $g(q)$, we obtain

$$
\begin{aligned}
g(z) & =\frac{1}{2 \pi} \int_{-\infty}^{\infty} d q \exp (i q z) g(q) \\
& =\frac{c_{1}}{2 \pi} \int_{-\infty}^{\infty} d q \frac{w(q) \exp \left(i q\left(z-z_{1}\right)\right)}{m_{1}+w(q)} .
\end{aligned}
$$

Note that in Eq. (28) $w(q)$ is an even function, and thus Eq. (28) can be written as

$$
g(z)=\frac{c_{1}}{\pi} \operatorname{Re} \int_{0}^{\infty} d q \frac{w(q) \exp \left(i q\left(z-z_{1}\right)\right)}{m_{1}+w(q)} .
$$

By choosing a contour on the upper right quadrant of the complex plane, we arrive at

$$
\begin{aligned}
g(z)= & \frac{c_{1}}{\pi} \operatorname{Re}\left[2 \pi i \sum_{n} \exp \left(i q_{n}\left(z-z_{1}\right)\right) R_{n}\right. \\
& \left.+\int_{0}^{\infty} d(i q) \frac{\exp \left(-q\left(z-z_{1}\right)\right) w(i q)}{m_{1}+w(i q)}\right],
\end{aligned}
$$

where $q_{n}$ is the $n$th pole in the first quadrant and $R_{n}$ the residue of the function $w(q) /\left[m_{1}+w(q)\right]$ at $q=q_{n}$. The poles $q_{n}$ are given by zero of the denominator in Eq. (29): $m_{1}+w\left(q_{n}\right)=0$. Let us denote the integral in Eq. (30) as $I$ which can be rewritten as

$$
I=-\frac{c_{1}}{\pi} \operatorname{Re} \int_{0}^{\infty} d(i q) \exp \left(-q\left(z-z_{1}\right)\right) \frac{1}{1+w(i q) / m_{1}} .
$$

At this point we are only interested in the asymptote of the density profile, i.e., the profile at large values of $z$. Thus we can first expand $w(q)$ [the Fourier transformation of $w(r)$ for $q>0$ ] about $q=0$,

$$
w(q)=\sum_{k=0}^{\infty} w_{k} q^{2 k}+\tilde{w}_{9} q^{9}+\tilde{w}_{3} q^{3} .
$$

We then substitute Eq. (32) into Eq. (31). Keeping only the first order term in the expansion about $q=0$ of the integrand, the integral $I$ becomes

$$
\begin{aligned}
I \approx & \frac{c_{1}}{\pi} \int_{0}^{\infty} d q \exp \left(-q\left(z-z_{1}\right)\right) \\
& \times\left[\left(1+w_{0} / m_{1}\right)^{-2} \frac{\tilde{w}_{3}}{m_{1}} q^{3}+O\left(q^{5}\right)\right] .
\end{aligned}
$$

The integral $I$ in the form of Eq. (33) leads to another key result of this work,

$$
g(z) \approx \frac{c_{1}}{\pi} \frac{m_{1} \tilde{w}_{3}}{\left(m_{1}+w_{0}\right)^{2}} \frac{6}{\left(z-z_{1}\right)^{4}}+O\left(\left(z-z_{1}\right)^{-6}\right),
$$

where $\quad m_{1}+w_{0}=(\partial \mu / \partial \rho)\left(\rho_{1}\right)=\left(\rho_{1}^{2} \chi_{1}\right)^{-1} \quad$ and $\quad \tilde{w}_{3}$ $=-2 \epsilon \pi \sigma^{6}$.

From Eq. (34) one can see that the leading term of the asymptote of $\Delta \rho_{1}(z)$ has the form

$$
\Delta \rho_{1}(z) \sim c_{1} \frac{f_{1}}{\left(z-z_{1}\right)^{3}},
$$

where $f_{1}=4 \epsilon \sigma^{6} m_{1} \rho_{1}^{4} \chi_{1}^{2}$. Similarly, one can derive the leading term of the asymptote of $\Delta \rho(z)$ in the liquid region, that is

$$
\Delta \rho_{2}(z) \sim-c_{2} \frac{f_{2}}{\left(z-z_{2}\right)^{3}},
$$

where $f_{2}=4 \epsilon \sigma^{6} m_{2} \rho_{2}^{4} \chi_{2}^{2}$. Note that $c_{1}$ and $c_{2}$ in Eqs. (35) and (36) are two constants which depend on the behavior of density profile at $z_{1}$ and $z_{2}$. However, they cannot be determined from Eqs. (35) and (36) because these two equations only give rise the asymptotic behavior of the density profile at large $|z|$ and thus cannot be used to describe the behavior of the profile at $z_{1}$ and $z_{2}$. On the other hand, the symmetry of the density profile assures that $c_{1}$ and $c_{2}$ should satisfy $c_{1}=h\left(\rho_{2}-\rho_{1}\right)$ and $c_{2}=-c_{1}$, where $h$ is an unknown parameter.

Having derived the asymptote for the density profile of Lennard-Jones [Eqs. (35) and (36)] we will introduce the patching model in order to derive an analytical expression for the density profile. Here, two models for the density profile are introduced.

\section{A. Model I}

In the first model we assume $z_{1}=z_{2}=\tilde{z}_{0}$. The density profile can then be expressed as

$$
\begin{aligned}
& \rho(z)=\rho_{1}+h\left(\rho_{2}-\rho_{1}\right) \frac{f_{1}}{\left(z-\tilde{z}_{0}\right)^{3}}, \quad z>\tilde{z}_{1}, \\
& \rho(z)=\rho_{2}+h\left(\rho_{2}-\rho_{1}\right) \frac{f_{2}}{\left(z-\tilde{z}_{0}\right)^{3}}, \quad z<\tilde{z}_{2}, \\
& \rho(z)=\tilde{a} z+\tilde{b}, \quad \tilde{z}_{2}<z<\tilde{z}_{1} .
\end{aligned}
$$

Here we have made an assumption that the behavior of the density profile in the small region between $\tilde{z}_{1}$ and $\tilde{z}_{2}$ can be described by a linear function. We argue that the linear function can be considered as the first two terms in the Taylor expansion of the density profile near $\tilde{z}_{0}$. In Eq. (37) there are 


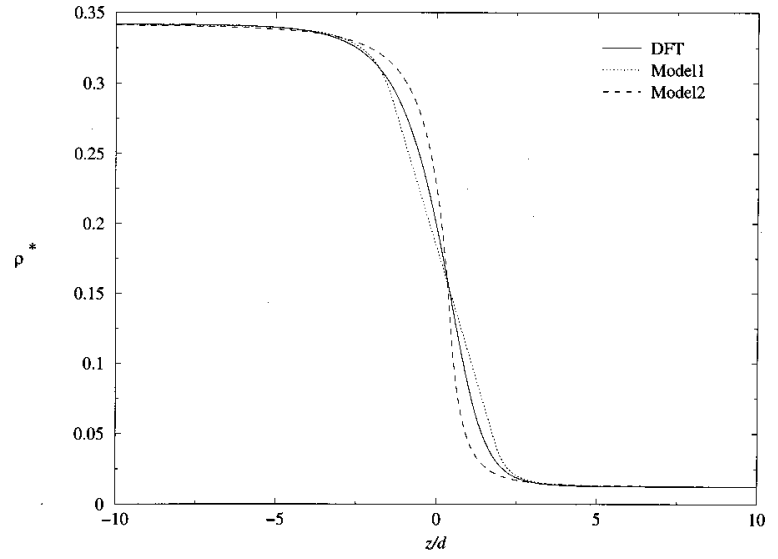

FIG. 1. The scaled density profile $\rho^{*}=(\pi / 6) \rho(z) d^{3}$ at temperature $T$ $=0.71 T_{c}$.

six unknown parameters: $\tilde{z}_{1}, \tilde{z}_{2}, \tilde{z}_{0}, \tilde{a}, \tilde{b}$, and $h$. However, only five equations are at hand; four represent the conditions of continuity of density profile and its first derivation at $\tilde{z}_{1}$ and $\tilde{z}_{2}$, which determine $\tilde{z}_{1}, \tilde{z}_{2}, \tilde{a}$, and $\tilde{b}$; the last one, Eq. (18), determines $\tilde{z}_{0}$. Expressions for the five parameters are summarized as the following:

$$
\begin{aligned}
& \tilde{z}_{0}=\frac{3}{4} 2^{2 / 3} h^{1 / 3}\left(f_{1}^{1 / 4}+f_{2}^{1 / 4}\right)^{1 / 3}\left(-f_{1}^{1 / 4}+f_{2}^{1 / 4}\right), \\
& \tilde{a}=-\frac{3}{8} 2^{1 / 3} h^{-1 / 3} \frac{\left(\rho_{2}-\rho_{1}\right)}{\left(f_{1}^{1 / 4}+f_{2}^{1 / 4}\right)^{4 / 3}}, \\
& \tilde{z}_{1}=\tilde{z}_{0}+\left(\frac{3 h\left(\rho_{1}-\rho_{2}\right) f_{1}}{\tilde{a}}\right)^{1 / 4}, \\
& \tilde{z}_{2}=\tilde{z}_{0}-\left(\frac{3 h\left(\rho_{1}-\rho_{2}\right) f_{2}}{\tilde{a}}\right)^{1 / 4}, \\
& \tilde{b}=\frac{\rho\left(\tilde{z}_{2}\right) \tilde{z}_{1}-\rho\left(\tilde{z}_{1}\right) \tilde{z}_{2}}{\tilde{z}_{1}-\tilde{z}_{2}} .
\end{aligned}
$$

Only $h$ remains an unknown parameter. We find that $h=1$ gives an excellent fit (see Fig. 1) to the numerical results of the DFT.

\section{B. Model II}

In the second model we assume that $z_{1}$ is in the liquid region whereas $z_{2}$ is in the vapor region, and $z_{1} \neq z_{2}$. In this case, the density profile is written as

$$
\begin{aligned}
& \rho(z)=\rho_{1}+h\left(\rho_{2}-\rho_{1}\right) \frac{f_{1}}{\left(z-z_{1}\right)^{3}}, \quad z>z_{0}, \\
& \rho(z)=\rho_{2}+h\left(\rho_{2}-\rho_{1}\right) \frac{f_{2}}{\left(z-z_{2}\right)^{3}}, \quad z<z_{0} .
\end{aligned}
$$

The four unknown parameters in Eq. (39) are $z_{1}, z_{2}, z_{0}$, and h. $z_{0}$ can be determined from Eq. (18); $z_{1}$ and $z_{2}$ from the conditions of continuity of the density profile and its derivation at $z_{0}$. Expressions of these three parameters are

$$
\begin{aligned}
& z_{0}=\frac{1}{2} h^{1 / 3}\left(f_{1}^{1 / 4}+f_{2}^{1 / 4}\right)^{1 / 3}\left(-f_{1}^{1 / 4}+f_{2}^{1 / 4}\right), \\
& z_{1}=z_{0}-h^{1 / 3}\left(f_{1}^{1 / 4}+f_{2}^{1 / 4}\right)^{1 / 3} f_{1}^{1 / 4},
\end{aligned}
$$

$$
z_{2}=z_{0}+h^{1 / 3}\left(f_{1}^{1 / 4}+f_{2}^{1 / 4}\right)^{1 / 3} f_{2}^{1 / 4} .
$$

As in the case of the first model, there is no equation to determine $h$. However, if $h=3$, Eq. (39) gives a density profile very close to the numerical one. Figure 1 shows the density profiles from both model I and II as well as that obtained by numerically solving Eq. (4). ${ }^{6}$ It can be seen that the differences in $\Delta \rho(z)$ only take place near the dividing surface and that the two models give, respectively, the upper and lower estimate for the density profile. In the next section, both models will be used to calculate the surface tension and the Tolman length.

\section{SURFACE TENSION AND THE TOLMAN LENGTH}

The main purpose of this work is to obtain analytical expressions for the surface tension of planar liquid-vapor interface and the Tolman length, given the analytical density profile of the patching model. To this end, we adopt the statistical mechanics formulas of Blokhuis and Bedeaux ${ }^{12}$ for the surface tension of planar vapor-liquid interface $\sigma_{\infty}$ and for the Tolman length $\delta_{\infty}$ under the random phase approximation,

$$
\begin{aligned}
\sigma_{\infty}= & \frac{\pi}{2} \int_{-\infty}^{\infty} d z \int_{0}^{\infty} d r r^{3} \frac{\partial w(r)}{\partial r} \int_{-1}^{1} d s\left(1-3 s^{2}\right) \\
& \times \rho(z) \rho(z+s r),
\end{aligned}
$$

and

$$
\begin{aligned}
\delta_{\infty}= & -\frac{\pi}{4 \sigma_{\infty}} \int_{-\infty}^{\infty} d z \int_{0}^{\infty} d r r^{3} \frac{\partial w(r)}{\partial r} \int_{-1}^{1} d s\left(1-3 s^{2}\right) \\
& \times(2 z+s r) \rho(z) \rho(z+s r) .
\end{aligned}
$$

In deriving Eq. (42), the equimolar dividing surface is located at $z=0$, which is also used in Eq. (18).

\section{A. Yukawa system}

For the Yukawa system, the integrals in Eqs. (41) and (42) can be carried out analytically when the density profile [Eq. (16)] is given. One can first obtain the surface tension

$$
\sigma_{\infty}=\left(\rho_{2}-\rho_{1}\right)^{2} \frac{\zeta_{1} \zeta_{2}}{\zeta_{1}+\zeta_{2}} \frac{\beta d}{2} l\left(\zeta_{1}, \zeta_{2}\right)
$$

where

$l\left(\zeta_{1}, \zeta_{2}\right)=\frac{\zeta_{1}^{2} \zeta_{2}+\zeta_{2}^{2} \zeta_{1}+4 \zeta_{1} \zeta_{2}+2 \zeta_{1}^{2}+2 \zeta_{2}^{2}+4 \zeta_{1}+4 \zeta_{2}+2}{2\left(\zeta_{1}+1\right)^{2}\left(\zeta_{2}+1\right)^{2}}$.

Function $l\left(\zeta_{1}, \zeta_{2}\right)$ changes slowly with increasing the temperature and approaches to the limiting value 1 as $T \rightarrow T_{c}\left(T_{c}\right.$ is the critical temperature). Thus the temperature dependence of $\sigma_{\infty}$ is mainly controlled by other terms in Eq. (43).

It is interesting to point out that the simple mean-field formula for surface tension ${ }^{4,13,20}$ is given by

$$
\sigma_{\infty}=2 m \int_{-\infty}^{\infty} d z\left(\frac{\partial \rho(z)}{\partial z}\right)^{2}
$$

where $m=(\pi / 15) \int_{0}^{\infty} d r r^{5}(\partial w(r) / \partial r)$, and for Yukawa system it leads to 


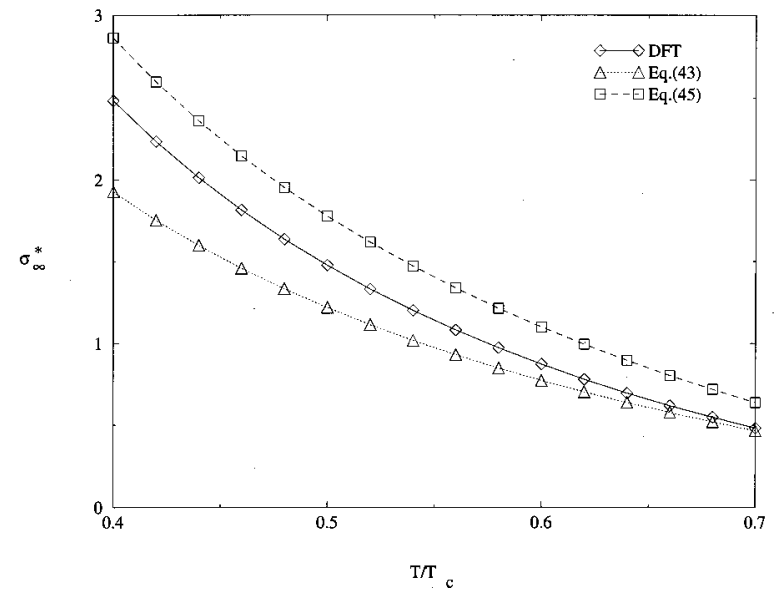

FIG. 2. The scaled surface tension of planar liquid-vapor interface $\sigma_{\infty}^{*}$ $=(\pi / 6)\left(d^{2} / k_{B} T\right) \sigma_{\infty}$ vs the reduced temperature $T / T_{c}$ for the Yukawa system.

$$
\sigma_{\infty}=\left(\rho_{2}-\rho_{1}\right)^{2} \frac{\zeta_{1} \zeta_{2}}{\zeta_{1}+\zeta_{2}} \frac{\beta d}{2}
$$

which would be the same as Eq. (43) if $l\left(\zeta_{1}, \zeta_{2}\right)=1$. Equation (45) was also derived under the square gradient approximation by Iwamatsu. ${ }^{16}$ As mentioned before, this approximation can give unphysical values of $\zeta$, e.g., $\zeta>1$ at low temperatures, leading to incorrect temperature dependence of surface tension.

In Fig. 2 the temperature dependence of surface tension is presented. The solid curve represents results of numerical calculation of density profile (exact) based on Eq. (41). ${ }^{6}$ The two dashed curves are obtained based on Eqs. (43) and (45). It can be seen that Eq. (45), and Eq. (43) in particular, gives quite good results of surface tension over a large range of temperatures.

Next, substituting the density profile (16) into Eq. (42) gives the Tolman length

$$
\delta_{\infty}=d \frac{\left(\zeta_{2}-\zeta_{1}\right)}{2 \zeta_{1} \zeta_{2}} \tilde{l}\left(\zeta_{1}, \zeta_{2}\right)
$$

where

$$
\begin{aligned}
& \tilde{l}\left(\zeta_{1}, \zeta_{2}\right) \\
& \quad=\frac{\left(\zeta_{1}+\zeta_{2}\right)\left(\zeta_{1}^{2}+\zeta_{2}^{2}+\zeta_{1} \zeta_{2}+3 \zeta_{1}+3 \zeta_{2}+3\right)-\zeta_{1} \zeta_{2}+1}{\left(\zeta_{1}+1\right)^{3}\left(\zeta_{2}+1\right)^{3} l\left(\zeta_{1}, \zeta_{2}\right)} .
\end{aligned}
$$

As $l\left(\zeta_{1}, \zeta_{2}\right)$, function $\tilde{l}\left(\zeta_{1}, \zeta_{2}\right)$ also changes slowly with increasing temperature and approaches to the limiting value 1 when $T \rightarrow T_{c}$. The temperature dependence of $\delta_{\infty}$ is thus mainly controlled by the terms before the function $\tilde{l}\left(\zeta_{1}, \zeta_{2}\right)$ in Eq. (46).

A similar expression of $\delta_{\infty}$ can also be derived from the mean-field formula ${ }^{13,14,20}$

$$
\delta_{\infty}=-\frac{2 m}{\sigma_{\infty}} \int_{-\infty}^{\infty} d z z\left(\frac{\partial \rho(z)}{\partial z}\right)^{2}
$$

which becomes (for Yukawa system)

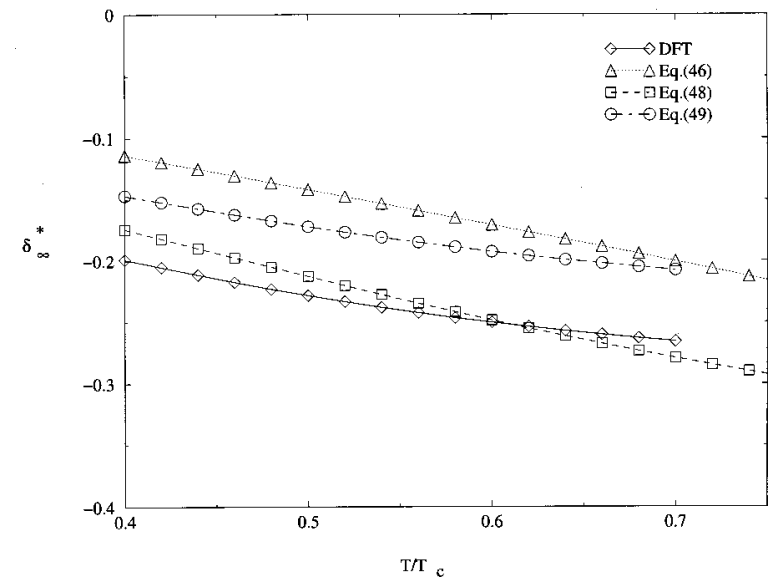

FIG. 3. The scaled Tolman length $\delta_{\infty}^{*}=\delta_{\infty} / d$ vs the reduced temperature $T / T_{c}$ for Yukawa system.

$$
\delta_{\infty}=d \frac{\left(\zeta_{2}-\zeta_{1}\right)}{2 \zeta_{1} \zeta_{2}}
$$

We note that Eq. (46) can be written in another way,

$$
\delta_{\infty}=-\frac{\sigma_{\infty}}{\left(\rho_{2}-\rho_{1}\right)^{2}}\left(k\left(\rho_{2}\right) \rho_{2}^{2} \chi_{2}-k\left(\rho_{1}\right) \rho_{1}^{2} \chi_{1}\right) \frac{l\left(\zeta_{1}, \zeta_{2}\right)}{\widetilde{l}\left(\zeta_{1} 0, \zeta_{2}\right)},
$$

where $k\left(\rho_{b}\right) \equiv\left(\partial \mu_{h} / \partial \rho\right)\left(\rho_{b}\right) \beta^{-1}$. This expression of $\delta_{\infty}$ is very similar to that obtained by Koga and Zeng. ${ }^{17}$ They found that $\delta_{\infty}$ is proportional to a quantity $\Delta_{\infty}=-\left[\sigma_{\infty} /\left(\rho_{2}\right.\right.$ $\left.\left.-\rho_{1}\right)^{2}\right]\left(\rho_{2}^{2} \chi_{2}-\rho_{1}^{2} \chi_{1}\right)$, but the coefficient of proportionality was unknown. Formula (49) differs from $\Delta_{\infty}$ by the function $k\left(\rho_{b}\right)$ and the factor $l / \tilde{l}$. The latter depends on the potential function $w(r)$.

In Fig. 3 the temperature dependence of the Tolman length $\delta_{\infty}$ is shown. The four curves correspond, respectively, to the numerical DFT calculations with Eq. (42), ${ }^{6} \mathrm{Eq}$. (46), Eq. (48), and Eq. (49) with numerical DFT values of $\sigma_{\infty}$. It can be seen that all curves lie close to each other. In particular, the shape and the bend of the curve [from Eq. (49)] is very similar to the curve from DFT calculations. All four curves show that the Tolman length is a small negative quantity with its absolute value weakly increasing with temperature.

\section{B. Lennard-Jones system}

To calculate the surface tension and the Tolman length for the Lennard-Jones system, two models of the density profile developed in the previous section are used. However, we found that the integrals in Eqs. (41) and (42) become so complicated that even for the simple density profiles, e.g., Eqs. (37) and (39), it is difficult to carry out the integrals analytically. Analytical expressions for surface tension and the Tolman length are possible only when the simple meanfield formulas, Eqs. (44) and (47), are employed. For model I with $h=1$, Eq. (44) gives

$$
\sigma_{\infty}=2^{1 / 3} \frac{9}{14} \frac{m\left(\rho_{2}-\rho_{1}\right)^{2}}{\left(f_{1}^{1 / 4}+f_{2}^{1 / 4}\right)^{4 / 3}},
$$


where $m=(144 / 35) 2^{5 / 6} \sigma^{5} \epsilon$; for model II with $h=3$, Eq. (44) gives

$$
\sigma_{\infty}=3^{-1 / 3} \frac{18}{7} \frac{m\left(\rho_{2}-\rho_{1}\right)^{2}}{\left(f_{1}^{1 / 4}+f_{2}^{1 / 4}\right)^{4 / 3}} .
$$

The two expressions of surface tension differ only by a factor. But given the fact that many assumptions and approximations are made for building up the density profiles, that the mean-field nature of the formulas (44), and that the application of Eq. (44) for the Lennard-Jones is not as good as for the Yukawa since all coefficients, except $m$, are divergent for the Lennard-Jones, the analytical expressions (50) and (51) should be considered merely as a simple estimation of $\sigma_{\infty}$.

In Fig. 4 the dependence of surface tension on temperature is presented; Fig. 4(a) shows results for model I and Fig. 4(b) for model II. It can be seen that $\sigma_{\infty}$ calculated (numerically) based on the two model density profiles and Eq. (41) are in very good agreement with those based on the exact density profile using formula (41). Figure 4(c) shows the temperature dependence of $\sigma_{\infty}$ given by

$$
\sigma_{\infty}=0.6 m \frac{\left(\rho_{2}-\rho_{1}\right)^{2}}{\left(f_{1}^{1 / 4}+f_{2}^{1 / 4}\right)^{4 / 3}},
$$

where the factor 0.6 is chosen since it gives the best fit to the numerical calculations. Not surprisingly, this formula gives better agreement of $\sigma_{\infty}$ compared with the DFT calculations.

Using the simple mean-field formula, analytical expressions for the Tolman length is also derived; for model I with $h=1$, Eq. (47) gives

$$
\delta_{\infty}=-\frac{1}{6} 2^{2 / 3}\left(f_{1}^{1 / 4}+f_{2}^{1 / 4}\right)^{1 / 3}\left(-f_{1}^{1 / 4}+f_{2}^{1 / 4}\right),
$$

and for model II with $h=3$ it gives

$$
\delta_{\infty}=-3^{-2 / 3}\left(f_{1}^{1 / 4}+f_{2}^{1 / 4}\right)^{1 / 3}\left(-f_{1}^{1 / 4}+f_{2}^{1 / 4}\right) .
$$

Combining Eq. (51) with (54), or Eq. (52) with (55) one can arrive at a formula for $\delta_{\infty}$ similar to Eq. (49),

$$
\begin{aligned}
\delta_{\infty}= & -\tilde{c} \frac{\sigma_{\infty}}{\left(\rho_{2}-\rho_{1}\right)^{2}}\left(k^{1 / 2}\left(\rho_{2}\right) \rho_{2}^{2} \chi_{2}\right. \\
& \left.-k^{1 / 2}\left(\rho_{1}\right) \rho_{1}^{2} \chi_{1}\right) \frac{\left(f_{1}^{1 / 4}+f_{2}^{1 / 4}\right)^{2 / 3}}{\sigma^{1 / 2}},
\end{aligned}
$$

where $\tilde{c}=(245 / 729)\left(3^{1 / 2} / 2^{1 / 4}\right)$ for model I and $(245 / 729)$ $\times\left(3^{1 / 2} / 2^{1 / 4}\right)\left(3^{2 / 3} / 4\right)$ for model II. Taking $\tilde{c}=0.85$ gives the best agreement of $\delta_{\infty}$ with the numerical calculations.

In Fig. 5 the temperature dependence of the Tolman length is presented; results for model I are in Fig. 5(a), and model II in Fig. 5(b). The four curves represent, respectively, the numerical DFT calculations with Eq. (42), ${ }^{6}$ numerical calculations using model density profiles with Eq. (42), calculations using Eq. (54) and Eq. (55). For the latter the DFT values of $\sigma_{\infty}$ were used. Results of $\delta_{\infty}$ based on Eq. (55) with $\tilde{c}=0.85$ are shown in Fig. 5(c). As in the case of the Yukawa, all calculations indicate that $\delta_{\infty}$ is a small negative quantity with its absolute value weakly increasing with temperature. It can be also seen that calculations of $\delta_{\infty}$ based on the model density profiles with Eq. (42) give correct shape
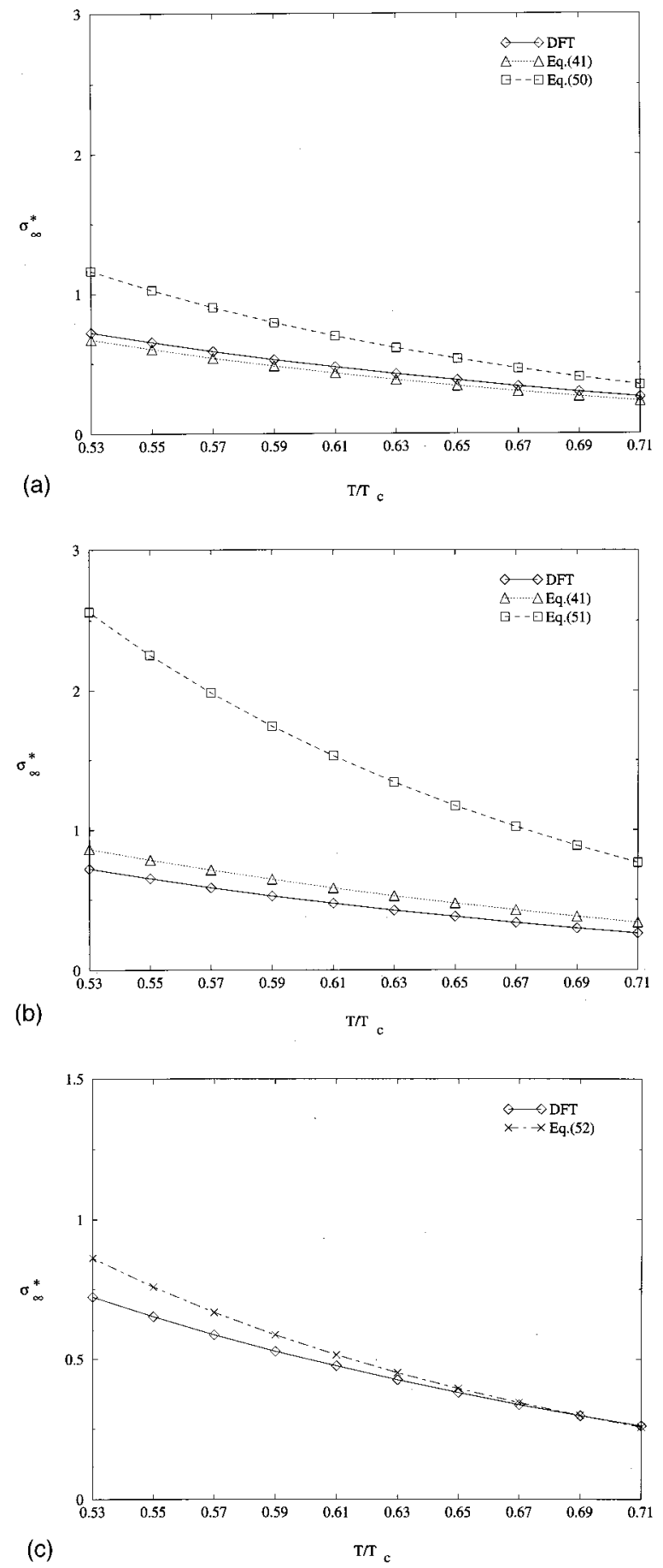

FIG. 4. The scaled surface tension of planar liquid-vapor interface $\sigma_{\infty}^{*}$ $=(\pi / 6)\left(d^{2} / k_{B} T\right) \sigma_{\infty}$ vs the reduced temperature $T / T_{c}$ for the Lennard-Jones system. Results based on (a) model I, (b) model II, and (c) Eq. (52).

and bend of the $\delta_{\infty}-T$ curve, and in particular, model II gives a better quantitative agreement with the numerical DFT results. The analytical expression (55) with $\tilde{c}=0.85$ gives the best quantitative description of the temperature dependence of the Tolman length for the Lennard-Jones.

\section{CONCLUSION}

A patching model for the density profiles based on the analytical expressions for the asymptote of the profiles is 

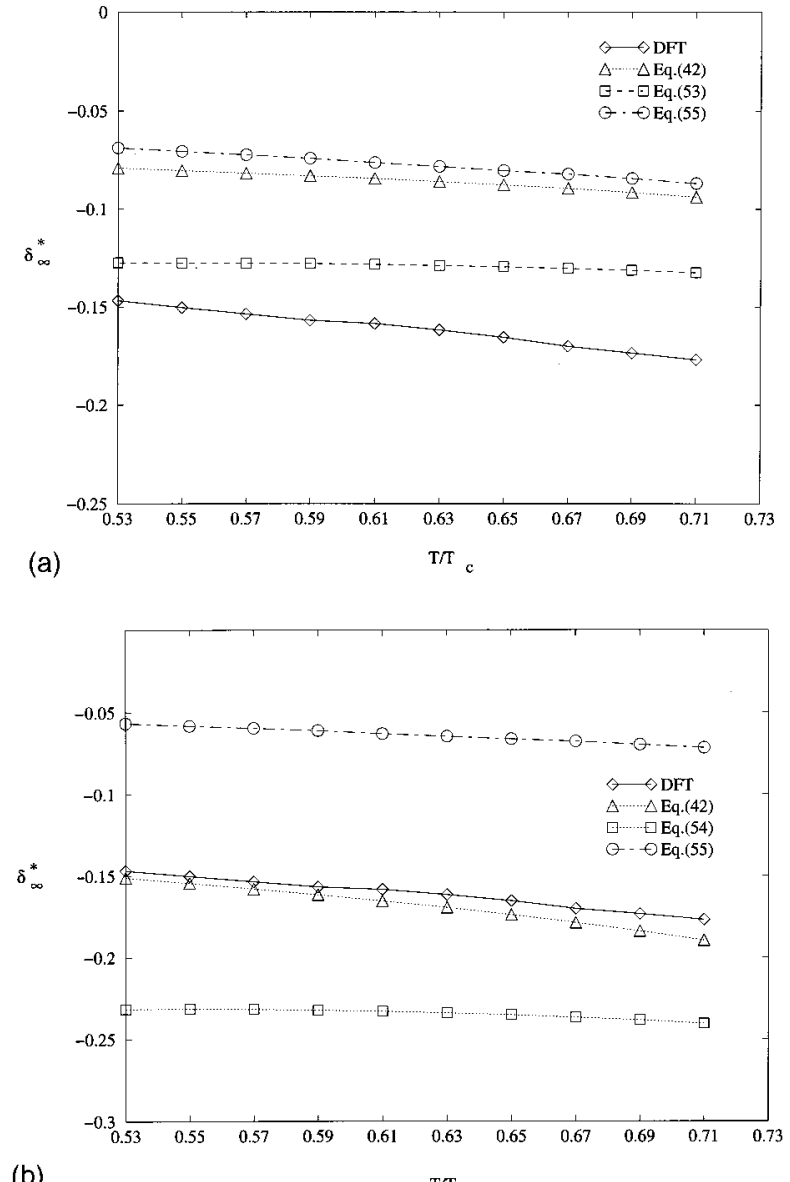

(b)

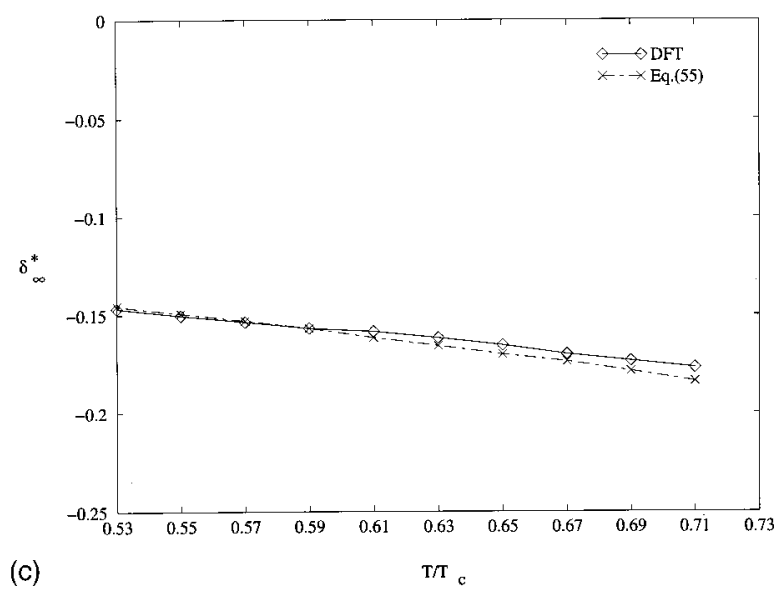

FIG. 5. The scaled Tolman length $\delta_{\infty}^{*}=\delta_{\infty} / d$ vs the reduced temperature $T / T_{c}$ for the Lennard-Jones system. Results based on (a) model I, (b) model II, (c) Eq. (55) with $\tilde{c}=0.85$.

developed. The model allows us to derive analytical expressions of the surface tension of the planar liquid-vapor interface and the Tolman length for both short-range Yukawa and long-range Lennard-Jones potentials. Despite many assumptions and simplifications, the resulting analytical expressions provide a good description of temperature dependence of the surface tension and the Tolman length over a large range of temperatures, compared with numerical DFT results.

As already found in many previous work, ${ }^{10,11,15,17,6}$ we also find the Tolman length is a small negative quantity with its absolute value weakly increasing with temperature. Based on the analytical expression of $\delta_{\infty}$ for the Yukawa [Eq. (49)] and for the Lennard-Jones [Eq. (55)] it is tempting to suggest a general formula for the Tolman length,

$$
\delta_{\infty} \sim-\frac{\sigma_{\infty}}{\left(\rho_{2}-\rho_{1}\right)^{2}}\left[N\left(\rho_{2}\right) \rho_{2}^{2} \chi_{2}-N\left(\rho_{1}\right) \rho_{1}^{2} \chi_{1}\right] \tilde{N}\left(\rho_{1}, \rho_{2}\right),
$$

where $N\left(\rho_{b}\right)$ and $\tilde{N}\left(\rho_{1}, \rho_{2}\right)$ are dimensionless function and dependent on the attractive part of intermolecular potential $w(r)$. Since values of both functions are always positive the sign of $\delta_{\infty}$ is essentially controlled by terms in bracket in Eq. (56). It seems that for all physical situations we have considered, $N\left(\rho_{2}\right) \rho_{2}^{2} \chi_{2}-N\left(\rho_{1}\right) \rho_{1}^{2} \chi_{1}$ is always greater than zero, and therefore $\delta_{\infty}<0$. Two other formulas of $\delta_{\infty}$, obtained recently by Iwamatsu ${ }^{16}$ and by Koga and Zeng, ${ }^{17}$ can be viewed as special cases of the general formula (56).

In this work, the free energy functional with the local density approximation is employed to derive the second direct correlation function. More sophisticated approximations such as the weighted density approximation (WDA) (Ref. 4) can certainly be used. To what extent the WDA will improve the surface tension and the Tolman length remains to be seen.

Finally, we point out that another way to obtain the Tolman length is via making approximation to the first curvature correction to the density profile. ${ }^{6}$ This correction can also be used to derive analytical expressions for the effective rigidity constant (the second curvature coefficient in the expansion of surface tension of droplet with small curvature). This will be a subject of future study. Indeed, both the Tolman length and the effective rigidity constant have been used to obtain the curvature dependence of surface tension for large droplets. $12,16,22,6$

\section{ACKNOWLEDGMENTS}

We are grateful to Professor Alexander Shchekin and Dr. Kenichiro Koga for useful discussions. X.C.Z. thanks the support by the National Science Foundation.

${ }^{1}$ R. C. Tolman, J. Chem. Phys. 17, 333 (1949).

${ }^{2}$ R. Becker and W. Döring, Ann. Phys. 24, 719 (1935).

${ }^{3}$ M. P. Allen and D. J. Tildesley, Computer Simulation of Liquids (Oxford University Press, New York, 1987).

${ }^{4}$ R. Evans, in Fundamentals of Inhomogeneous Fluids, edited by D. Henderson (Wiley, New York, 1992).

${ }^{5}$ A. I. Rusanov and E. N. Brodskaya, J. Colloid Interface Sci. 62, 542 (1977).

${ }^{6}$ T. V. Bykov and A. K. Shchekin, Inorg. Mater. 35, 641 (1999).

${ }^{7}$ T. V. Bykov and A. K. Shchekin, Colloid J. USSR 61, 144 (1999).

${ }^{8}$ M. J. Nijmeijer, C. Bruin, A. B. van Woerkom, and A. F. Bakker, J. Chem. Phys. 96, 565 (1992).

${ }^{9}$ M. J. Haye and C. Bruin, J. Chem. Phys. 100, 556 (1994).

${ }^{10}$ V. Talanquer and D. W. Oxtoby, J. Phys. Chem. 99, 2865 (1995).

${ }^{11}$ K. Koga, X. C. Zeng, and A. K. Shchekin, J. Chem. Phys. 109, 4063 (1998).

${ }^{12}$ E. M. Blokhuis and D. Bedeaux, Physica A 184, 42 (1992).

${ }^{13}$ E. M. Blokhuis and D. Bedeaux, Mol. Phys. 80, 705 (1993).

${ }^{14}$ M. P. A. Fisher and M. Wortis, Phys. Rev. B 29, 6252 (1984).

${ }^{15}$ A. E. van Giessen, E. M. Blokhuis, and D. J. Bukman, J. Chem. Phys. 108, 1148 (1998).

${ }^{16}$ M. Iwamatsu, J. Phys.: Condens. Matter 5, 7537 (1993); 6, L173 (1994).

${ }^{17}$ K. Koga and X. C. Zeng, J. Chem. Phys. 110, 3466 (1999). 
${ }^{18}$ X. C. Zeng and D. W. Oxtoby, J. Chem. Phys. 94, 4472 (1991).

${ }^{19}$ J. D. Weeks, D. Chandler, and H. C. Andersen, J. Chem. Phys. 54, 5237 (1971).

${ }^{20}$ E. M. Blokhuis and D. Bedeaux, Heterog. Chem. Rev. 1, 55 (1994).
${ }^{21}$ R. J. Leote de Carvalho, R. Evans, D. C. Hoyle, and J. R. Henderson, J. Phys.: Condens. Matter 6, 9275 (1994).

${ }^{22}$ V. G. Baidakov and G. Sh. Boltachev, Russ. J. Phys. Chem. 69, 515 (1995); Phys. Rev. E 59, 469 (1999). 